\title{
Oncological Outcome and Patient Satisfaction in Skin-Sparing Mastectomy and Immediate Breast Reconstruction
}

Mansour Mohammed Kabbash ${ }^{1}$, Anwar Abdu El Shenawy ${ }^{2}$, Mohamed Mahmoud Eloteify ${ }^{3}$, Sherief El Prince Sayed ${ }^{2}$, Khaled Abdeen Talha Suleiman ${ }^{1 *}$

${ }^{1}$ Department of General Surgery, ${ }^{2}$ Department of Oncosurgery,

${ }^{3}$ Department of Plastic Surgery, Faculty of Medicine, Aswan University

*Corresponding author: Khaled Abdeen Talha Suleiman, Mobile: (+20) 01002028155, E-Mail: khaledsuleiman@ymail.com

\begin{abstract} estimated new cases annually over a 20 -year span. breast cancer. University Hospitals, between 2015 and 2019. follow up might be a drawback of obtaining a solid conclusion.

\section{INTRODUCTION}

Approximately 1 in 8 women (13\%) will be diagnosed with invasive breast cancer in their lifetime and 1 in 39 women (3\%) will die from breast cancer ${ }^{(\mathbf{1})}$.

In Egypt, Data reported by Gharbia population based cancer registry (2001) indicated that breast cancer ranked first among women (37.6\%), with an age standardized rate of 49.6/100000.On the other hand, carcinoma of the men breast was only $0.5 \%$ (2).
\end{abstract}

Background: Breast cancer represents $10 \%$ of all cancers diagnosed worldwide annually and constitute $22 \%$ of all new cancers in women. The burden of breast cancer has increased steadily, almost doubling, in terms of

Objective: This study was aimed to evaluate the outcome on oncological standard and patient satisfaction on the aesthetic side with skin-sparing mastectomy and immediate breast reconstruction for patients with early

Patients and methods: this study included 80 female patients with breast cancer, 60 cases were operated upon at National Cancer Institute (NCI), Cairo University, 20 cases were operated upon at Oncosurgery Unit, Aswan

Results: In the current study, all patients were closely followed up to detect any signs of local recurrence (LR). The duration of postoperative follow up ranged from 24 to 48 months with a mean of 31.55 months. Only 1 patient $(1.2 \%)$ showed signs of local recurrence after 1 year of surgery. This condition was not associated with any sign of regional or systemic recurrence. This low incidence of local recurrence may be attributed to the early stage of the disease at the time of surgical intervention, the mastectomy procedure itself and the justified administration of neoadjuvant and adjuvant therapy to the studied patients. However, a relatively short period of

Conclusions: It could be concluded that skin sparing mastectomy and Immediate breast reconstruction is a technically feasible and oncologically safe procedure. Accepted cosmetic result was attained in most of patients. Keywords: Breast cancer, Oncological outcome, Skin-sparing mastectomy, Immediate breast reconstruction.

The overarching principle guiding surgical management of women with breast cancer remains the oncological safety. The mainstay of satisfactory local control continues to be adequate clearance of the primary tumor and involved axillary lymph nodes ${ }^{(3)}$.

Improvements in understanding of tumor biology have enabled the risk of loco-regional recurrence and distant events to be further reduced by adjuvant, or neo-adjuvant, radiotherapy and systemic treatments. In keeping with this, breast conserving therapy has become well established as the treatment of choice for most women with early breast cancer ${ }^{(4)}$.

However, approximately one-third of women still undergo mastectomy, either due to patient preference or in cases where breast conservation is not oncologically or aesthetically compatible with the size or distribution of disease ${ }^{(5)}$.

Skin sparing mastectomy involves the enbloc removal of all glandular tissue including the nipple-areola complex and in some cases adjacent biopsy scars and skin overlying superficial tumours. In contrast to conventional mastectomy, there is maximal preservation of the remaining breast skin envelope and infra-mammary fold that facilitate immediate breast reconstruction with autologous tissue and/or prosthetic implants by utilizing the native skin envelope to optimize the contour, texture, colour and scarring of the reconstructed breast ${ }^{\left({ }^{6}\right.}$.

This approach combines the ablative and reconstructive components of the surgical intervention, offering a single-stage procedure which is likely to be popular with patients in terms of hospital admissions, return to employment and elimination of the postmastectomy prereconstruction period ${ }^{(7)}$.

The aesthetic advantages of skin sparing mastectomy have been tempered to some degree by concerns regarding oncological safety. In comparison with conventional mastectomy, the complete excision of glandular tissue during skin sparing mastectomy can be technically more demanding ${ }^{(8)}$. 
Skin sparing mastectomy can facilitate immediate breast reconstruction by the advantages in contour, colour, texture and scarring associated with preservation of the native skin envelope. This approach can also reduce the need for contra-lateral adjustment in order to achieve symmetry ${ }^{(9)}$.

Most women who will undergo skin sparing mastectomy and immediate breast reconstruction for early-stage breast cancer will not require postmastectomy radiotherapy. However, postmastectomy radiotherapy has been shown to reduce loco-regional recurrence and improve survival for patients with three or more involved regional lymph nodes or tumors $>5 \mathrm{~cm}^{(\mathbf{1 0})}$.

Several variations of the standard skin sparing mastectomy have recently been reported, including the nipple sparing mastectomy. Preservation of the nipple areola complex offers aesthetic advantages and eliminates the need for nipple reconstruction associated with standard skin sparing mastectomy. Oncological concerns regarding the risk of occult nipple areola complex involvement have been assuaged to some extent by several recent studies (11).

This study was aimed to evaluate the outcome on oncological standard and patient satisfaction on the aesthetic side with skin-sparing mastectomy and immediate breast reconstruction for patients with early breast cancer.

\section{PATIENTS AND METHODS}

This study included a total of 80 female patients with breast cancer, 60 cases were operated upon at National Cancer Institute (NCI), Cairo University and 20 cases were operated upon at oncosurgery Unit, Aswan University Hospitals, between 2015 and 2019.

\section{Ethical approval:}

\section{Approval of the ethical committee of Aswan} university was obtained. Written informed consent of all the subjects was obtained, explaining that the study is a research, and declared the details of the procedure and the anticipated benefits and complications.

Patient's age ranged from 20 to 50 years. they underwent skin sparing mastectomy with formal axillary clearance through a circum-areolar incision with lateral extension and immediate breast reconstruction to fill the skin envelop by:

1. Latissimus dorsi myocutaneous flap (LDMF) with implant In 32 patients.

2. Latissimus dorsi myocutaneous flap (LDMF) without implant In 24 patients.

3. Transverse rectus abdominis myocutaneous flap (TRAM) in 8 patients.
4. Subpectoral implant in 16 pateints.

All patients were subjected to:

- Thorough clinical examination of both breasts and axillae as regards:

- Breast lump

- Skin affection or changes

- Nipple discharge

- Axillary lymph nodal enlargement

- Laboratory investigations: such as.

- Complete blood count (CBC).

- Coagulation profile.

- Liver and kidney function tests.

- Plasma sugar assessment.

- Radiological investigations: such as

- Bilateral mammography.

- Ultrasonography of the breast and axilla on both sides.

- Routine metastatic work up: including

- Plain X ray chest.

- Abdominal ultrasonography.

- Bone scan if indicated.

The diagnosis of breast cancer was histologically proven by fine-needle aspiration cytology or core cut biopsy.

Pre-operative marking was done in the morning of the surgery after patient counseling and consenting, care was taken to select the most suitable procedure to fit each patient individually taking into consideration post-operative adjuvant treatment and medical comorbidities and body built.

Medical photography was preformed pre- and post-operatively and a scoring system for subjective assessment of the final cosmetic outcome was used.

Drawings included a circum-areolar incision and the lateral extension line, and the footprint of the breast was also outlined (the breast "footprint", is the outline that the breast makes on the chest wall) along with the infra- mammary fold.

A skin ellipse was designed over the donor site (on the back for the LD and lower abdomen for the TRAM flaps), and a disk was drawn corresponding to the diameter of the areola, and was centered on the flap to replace the skin of the NAC.

The incision was carried out after induction of general anesthesia with the patient placed supine on the operating table. Removal of the NAC along with the breast tissue was carried out through elevation of the skin flaps in the same planes as the NSSM. Care was taken during traction so as not to devitalize the native skin envelope and traction on the skin of the NAC was done to manipulate the 
specimen for adequate exposure. Dissection of the lower flap was not carried out beyond the inframammary fold, so as not to go beyond the breast footprint.

The excised breast was volumetrically assessed and histopathologically examined with special concern to the subareolar region and the distance between the tumor and the nipple-areola complex.

Immediate breast reconstruction was performed using different techniques to fill in the skin envelope. The reconstruction method was determined by the surgical oncology and the aesthetic surgery team according to anticipated adjuvant postoperative radiotherapy, body habitus, size of reconstructed breast and contralateral match, availability of flap donor sites, any co-existing co morbidities, previous abdominal surgeries and to patient's preference and acceptance for the reconstructive procedure.

Staging of the tumor was done in addition to assessment of hormonal receptors status (ER/PR), HER-2, tumor size, tumor characteristics, lymph nodal status and histological grading.

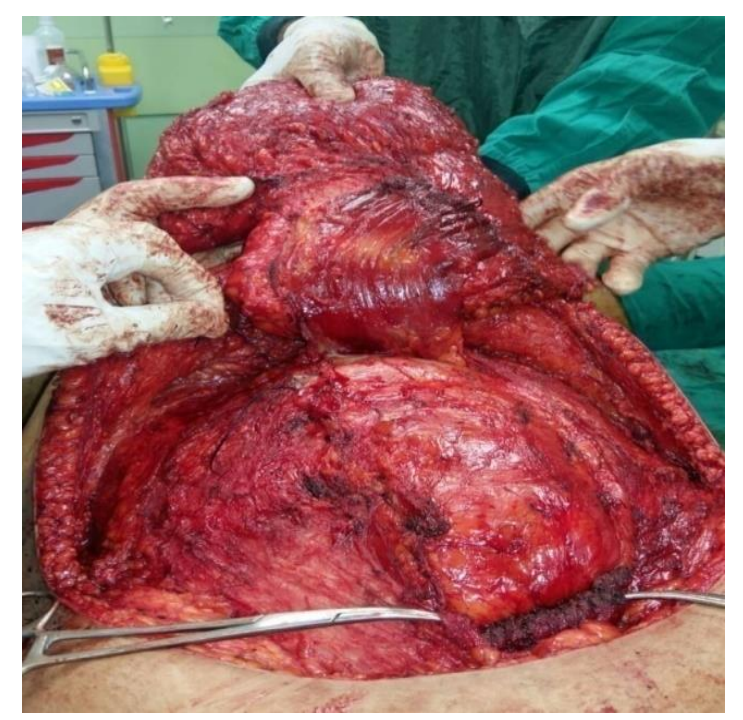

Figure (1): Transverse rectus abdominis myocutaneous flap.

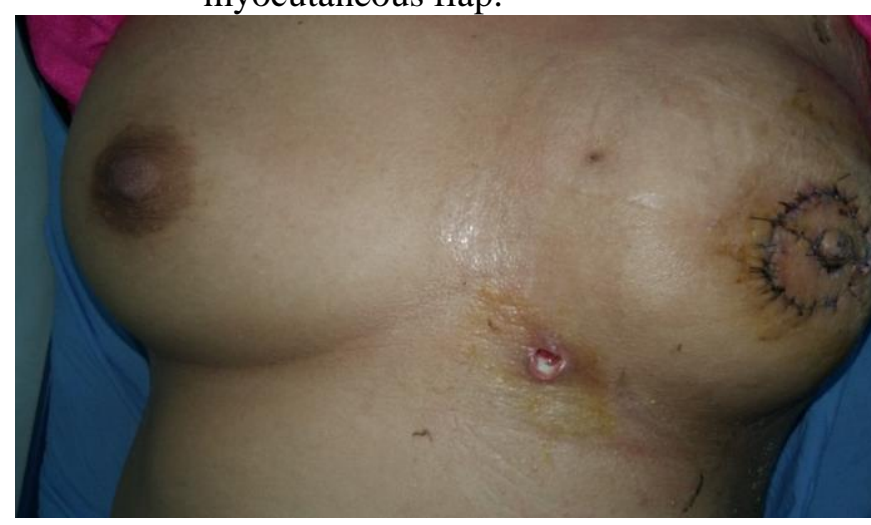

Figure (2): Extended LD flap reconstruction.

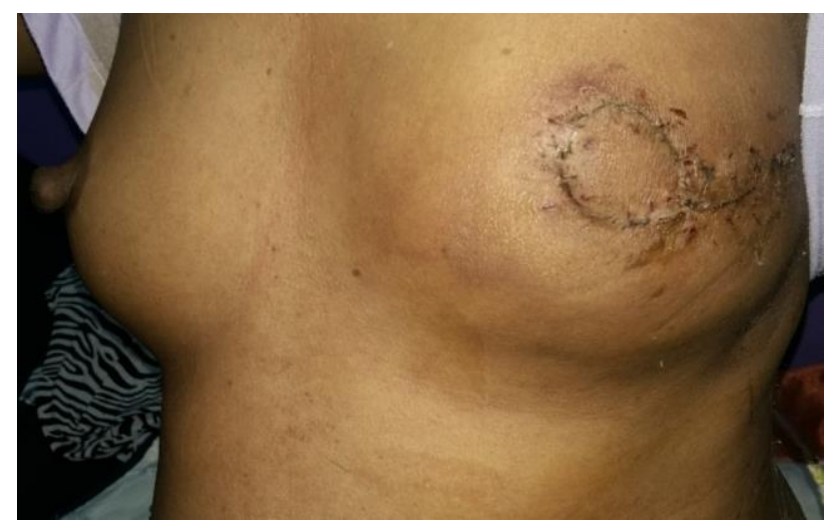

Figure (3): Reconstruction using an extended LD flap augmented by an implant placed within the LD pocket over the pectoralis muscle.

Adjuvant systemic chemotherapy, radiotherapy and hormonal therapy: was planned and administered according to histopathological examination, lymph nodal status and hormonal receptor status.

All patients were followed up starting 4-6 weeks after the operation. The duration of postoperative follow up ranged from 24 month postoperatively up to 48 month with a mean of 31.55 month of follow up, all patients were subjected to clinical examination every three months, ultrasonography of both breasts and axillae biannually and mammography every year aiming for:

a- Detection of any postoperative complication such as; infection, thrombosis, hematoma, flap necrosis.

b- Detection of any sign of local, locoregional or systemic recurrence.

\section{Data Management and Analysis:}

The collected data was revised, coded, tabulated and introduced to a PC using Statistical package for Social Science (SPSS 25). Data was presented and suitable analysis was done according to the type of data obtained for each parameter.

\section{Descriptive statistics:}

Mean, Standard deviation $( \pm$ SD) and range for parametric numerical data, while Median and Interquartile range (IQR) for non-parametric numerical data. Frequency and percentage of nonnumerical data.

\section{Statistics Analysis}

Chi-Square test was used to examine the relationship between two qualitative variables. Fisher's exact test was used to examine the relationship between two qualitative variables when the expected count is less than 5 in more than $20 \%$ of cells. 


\section{$P$ - value: level of significance}

$-\mathrm{P}>0.05$ : Non significant (NS).

$-\mathrm{P}<0.05$ : Significant $(\mathrm{S})$.

$-\mathrm{P}<0.01$ : Highly significant (HS).

\section{RESULTS}

A total of 80 female patients with early breast cancer matching with the inclusion criteria for skin sparing mastectomy and immediate breast reconstruction were included in our study. All had SSM and IBR, patient's age ranged from 20 to 50 years.

60 cases were operated upon at NCI (national cancer institute, Cairo university), 20 cases were operated upon at Aswan university hospitals Oncosurgery Unit between 2015 and 2019.

Table (1): Type of breast reconstruction procedure used for all cases included in our study.

\begin{tabular}{|c|c|c|c|}
\hline \multicolumn{2}{|c|}{} & $\mathrm{N}$ & $\%$ \\
\hline \multirow{4}{*}{$\begin{array}{c}\text { Reconstru } \\
\text { ction } \\
\text { procedure }\end{array}$} & LDMF with implant & 32 & $40.0 \%$ \\
\cline { 2 - 4 } & LDMF without implant & 24 & $30.0 \%$ \\
\cline { 2 - 4 } & Sub pectoral implant & 16 & $20.0 \%$ \\
\cline { 2 - 4 } & TRAM flap & 8 & $10.0 \%$ \\
\hline
\end{tabular}

Pre-operative data for all cases in our study were collected and the results.

\section{Pathological diagnosis}

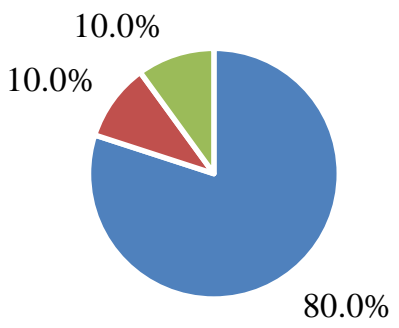

- Invasive duct carcinoma

- DCIS with diffuse micro calcification

- Pagets disease of nipple

Figure (4): Pathological diagnosis for all cases.

In our study, post-operative morbidities were in the form of donor-site seromas, superficial skin gangrene, local skin necrosis and gangrene of edges of skin and local recurrence.

Table (2): Post-operative complications.

\begin{tabular}{|c|c|c|c|}
\hline \multirow{2}{*}{ Donor site seroma } & & $\mathrm{N}$ & $\%$ \\
\cline { 2 - 4 } & No & 57 & $71.3 \%$ \\
\hline \multirow{2}{*}{$\begin{array}{c}\text { Superficial skin } \\
\text { gangrene of the flap }\end{array}$} & Yes & 23 & $28.8 \%$ \\
\cline { 2 - 4 } & No & 74 & $92.5 \%$ \\
\hline \multirow{2}{*}{ Local skin necrosis } & Yes & 6 & $7.5 \%$ \\
\cline { 2 - 4 } & No & 72 & $90.0 \%$ \\
\hline \multirow{2}{*}{ Local recurrence } & Yes & 8 & $10.0 \%$ \\
\cline { 2 - 4 } & No & 1 & $98.8 \%$ \\
\hline
\end{tabular}

Donor-site seromas found in 23 patients ( $28.8 \%$ of cases) in spite of the use of closed suction drains.

In the current study, all patients were closely followed up to detect any signs of local recurrence (LR). The duration of postoperative follow up ranged from 24 months up to 48 months with a mean of 31.55 month of follow up. Only 1 patient (1.3\%) showed signs of local recurrence after 1 year of surgery.

\section{Comparison analysis:}

Pre-operative data collected as regard BMI, co-existing co-morbidities and breast size and results. 
Table (3): Pre-operative data collected and comparison between the procedures used for reconstruction for all cases.

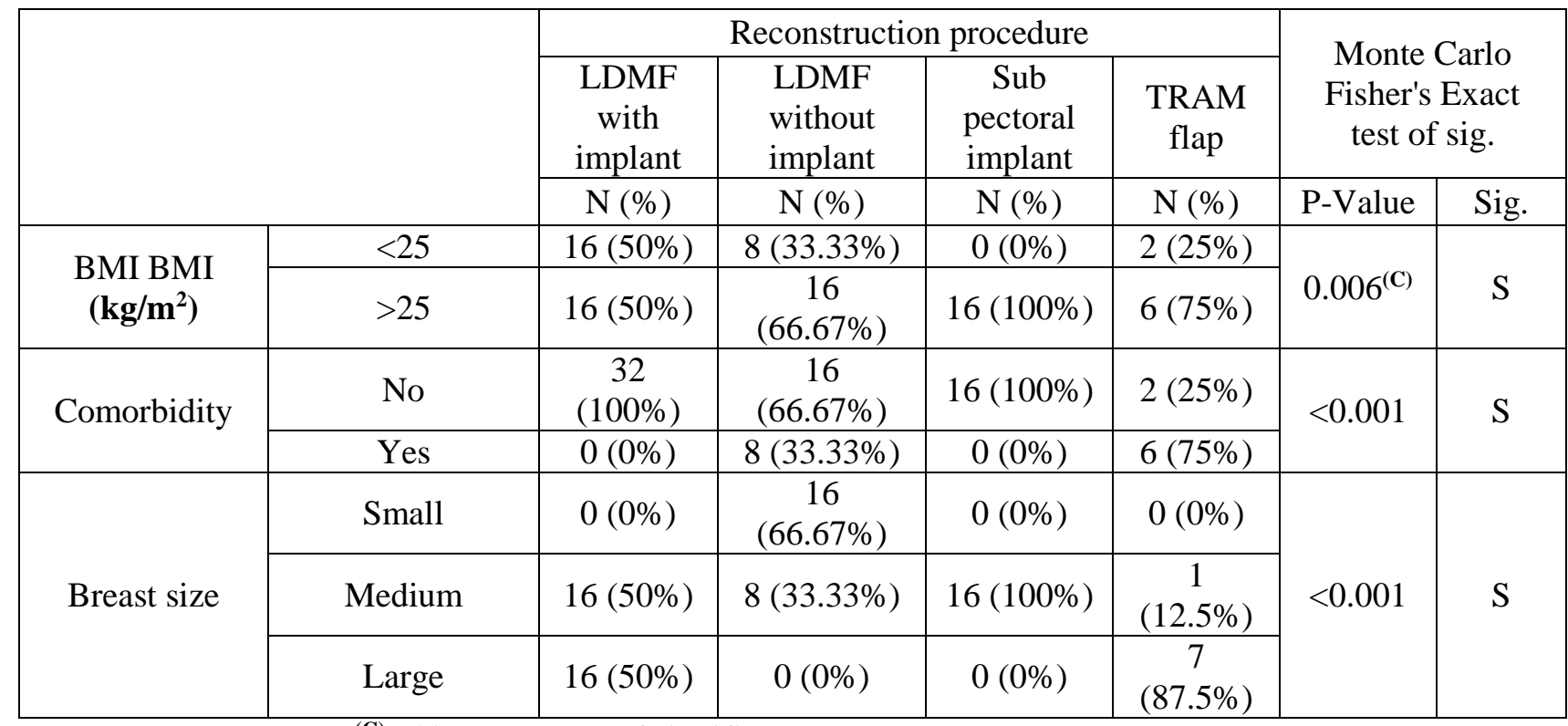

${ }^{(\mathrm{C})}$ Chi-Square test of significance.

In this study, assessment of the aesthetic results and patient's contentment was done through patient's questionnaire by the surgical team themselves involving a number of subjective evaluations of the reconstructed breast based on psychological satisfaction, Shape and contour of the reconstructed breast and contralateral match. The patients were classified according to their psychological satisfaction about the reconstructive procedure into 4 groups ranging from extremely satisfied, satisfied, less satisfied and finally dissatisfied.

Table (4): Post-operative data collected and comparison between the procedures used for reconstruction for all cases.

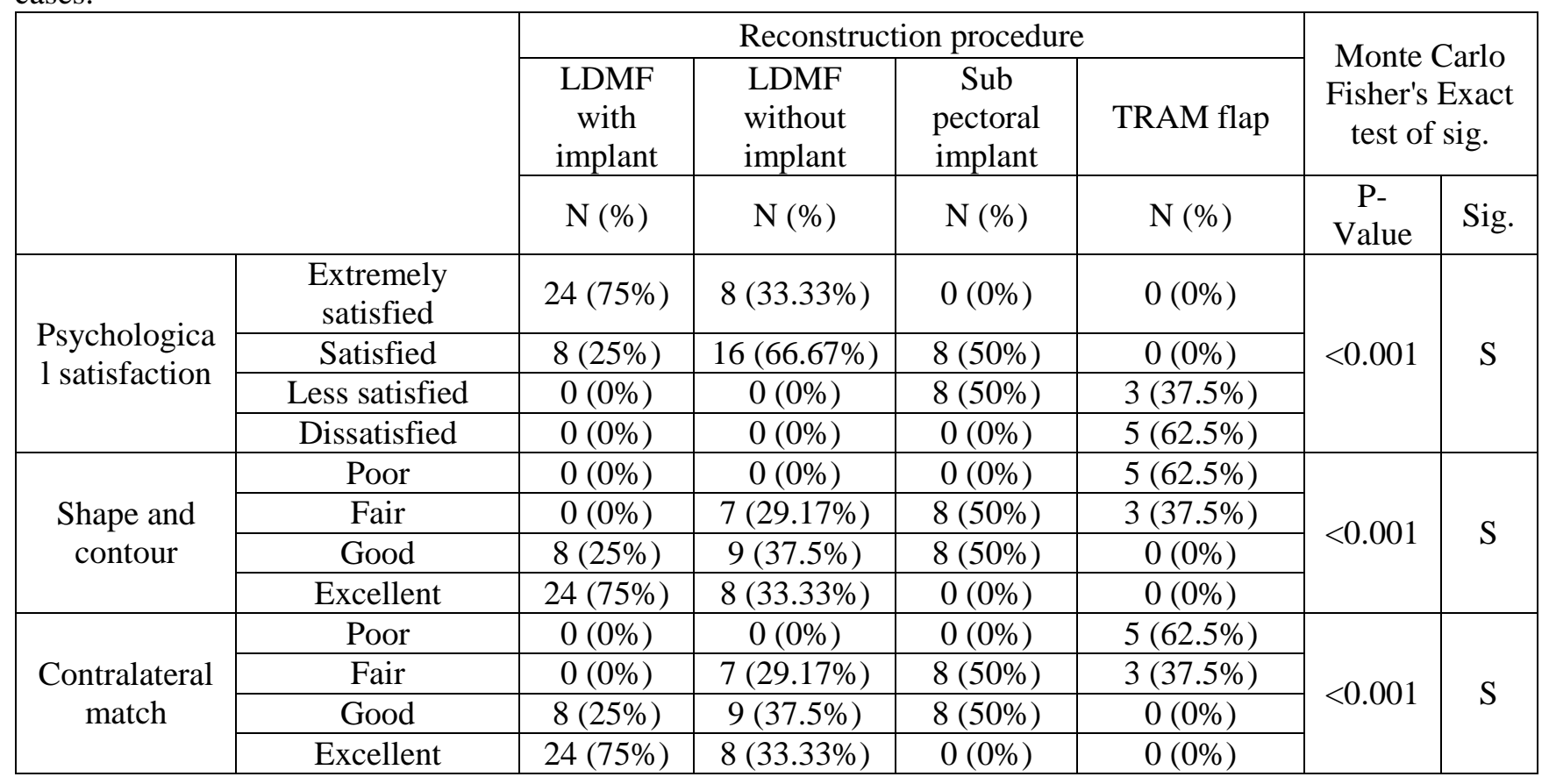

Post-operative morbidities were in the form of donor-site seromas, superficial skin gangrene, local skin necrosis and gangrene of edges of skin and local recurrence, data collected, compared between the procedures used for reconstruction for all cases and results. 
Table (5): Post-operative morbidities and comparison between the procedures used for reconstruction for all cases.

\begin{tabular}{|c|c|c|c|c|c|c|c|}
\hline \multicolumn{6}{|c|}{ Reconstruction procedure } & \multirow{2}{*}{\multicolumn{2}{|c|}{$\begin{array}{l}\text { Monte Carlo } \\
\text { Fisher's Exact } \\
\text { test of sig. }\end{array}$}} \\
\hline & & \multirow{2}{*}{$\begin{array}{c}\begin{array}{c}\text { LDMF } \\
\text { with } \\
\text { implant }\end{array} \\
\mathrm{N}(\%)\end{array}$} & \multirow{2}{*}{$\begin{array}{c}\begin{array}{c}\text { LDMF } \\
\text { without } \\
\text { implant }\end{array} \\
\mathrm{N}(\%)\end{array}$} & \multirow{2}{*}{$\begin{array}{c}\text { Sub pectoral } \\
\text { implant }\end{array}$} & \multirow{2}{*}{$\begin{array}{c}\begin{array}{c}\text { TRAM } \\
\text { flap }\end{array} \\
\mathrm{N}(\%)\end{array}$} & & \\
\hline & & & & & & $\begin{array}{c}\mathrm{P}- \\
\text { Value }\end{array}$ & Sig. \\
\hline \multirow{2}{*}{$\begin{array}{c}\text { Donor site } \\
\text { seroma }\end{array}$} & No & $32(100 \%)$ & $16(66.67 \%)$ & $8(50 \%)$ & $1(12.5 \%)$ & \multirow{2}{*}{$<0.001$} & \multirow{2}{*}{ S } \\
\hline & Yes & $0(0 \%)$ & $8(33.33 \%)$ & $8(50 \%)$ & $7(87.5 \%)$ & & \\
\hline \multirow{2}{*}{$\begin{array}{l}\text { Superficial skin } \\
\text { gangrene of } \\
\text { flap } \\
\end{array}$} & No & $32(100 \%)$ & $24(100 \%)$ & $16(100 \%)$ & $2(25 \%)$ & \multirow{2}{*}{$<0.001$} & \multirow[b]{2}{*}{ S } \\
\hline & Yes & $0(0 \%)$ & $0(0 \%)$ & $0(0 \%)$ & $6(75 \%)$ & & \\
\hline \multirow{2}{*}{$\begin{array}{c}\text { Local skin } \\
\text { necrosis }\end{array}$} & No & $32(100 \%)$ & $16(66.67 \%)$ & $16(100 \%)$ & $8(100 \%)$ & \multirow{2}{*}{$<0.001$} & \multirow{2}{*}{$S$} \\
\hline & Yes & $0(0 \%)$ & $8(33.33 \%)$ & $0(0 \%)$ & $0(0 \%)$ & & \\
\hline \multirow{2}{*}{$\begin{array}{c}\text { Local } \\
\text { recurrence }\end{array}$} & No & $32(100 \%)$ & $24(100 \%)$ & $15(93.75 \%)$ & $8(100 \%)$ & \multirow{2}{*}{0.304} & \multirow{2}{*}{ NS } \\
\hline & Yes & $0(0 \%)$ & $0(0 \%)$ & $1(6.25 \%)$ & $0(0 \%)$ & & \\
\hline
\end{tabular}

\section{DISCUSSION}

A total of 80 female patients with early breast cancer matching with the inclusion criteria for skin sparing mastectomy and immediate breast reconstruction were included in our study. They were subjected to thorough clinical examination, laboratory investigations and radiological assessment; all had SSM and IBR, patient's age ranged from 20 to 50 years, this may explain why those relatively young patients were well motivated to have IBR following SSM.

In their retrospective comparative study, Kinoshita et al studied 73 patients having SSM and IBR, the mean age for them was 47.09. \pm 0 (31-71 years) ${ }^{(\mathbf{1 2})}$.

In another series, Romics et al. studied 207 patients who were subjected to SSM and IBR, their median age was 49 (range 26-68). The median age of patients in the latter two studies was comparable to the median age of patients in our study, although, the upper limit for age in our study was remarkably lower than their upper limits of age. It was found that age of patients has no value in the final assessment of either the oncologic safety or the aesthetic outcome of the procedure ${ }^{(13)}$.

In the current study, patients were classified according to TNM classification into 3 groups as follow: 8 patients $(10 \%)$ stage 0,44 patients (55\%) stage I, 28 patients (35\%) stage $2 \mathrm{~A}$.

In Kinoshita's retrospective study, the SSM group were: stage O (11 patients), stage I (25 patients), stage IIA (28 patients) and finally stage IIB (9 patients) ${ }^{(\mathbf{1 2})}$.

In another prospective observational study by Reefy and her colleagues, the 137 cases studied (117 patient with unilateral lesion and 10 patients with bilateral lesions) were classified to be: 25 patients Tis, 63 patients T1, 41 patients T2, 1 patient as T3, and lastly, 7 cases showed benign postoperative histopathological findings ${ }^{(5)}$.

In our study, post-operative morbidities were in the form of: donor-site seromas found in 23 patients (28.8\% of cases), in spite of the use of closed suction drains, superficial skin gangrene reported in 6 patients $(7.5 \%)$ managed by debridement and daily dressings till spontaneous healing with resultant delay in the initiation of adjuvant chemo and radiotherapy for 2 month. Finally, 8 cases $(10 \%)$ had local necrosis and gangrene of edges of skin envelope necessitating removal of implants; this was responsible for a postoperative delay in conduction of adjuvant treatment for about 3 months. Patient's related factors including age, body habitus and co-existing co-morbidities were found to be insignificant in the occurrence of post-operative comorbidities. None of our patients were smoking.

In a series of 127 women who had SSM and IBR by Reefy $\boldsymbol{e} \boldsymbol{t} \boldsymbol{a l}$. they observed infection requiring implant removal in 2 patients and 1 patient developed marginal ischemia of skin envelope which was treated conservatively. All their patients who underwent LD flap reconstruction developed donor-site seroma which was managed conservatively. Chemotherapy was delayed in only one patient for 2 weeks due to infection. Six patients had previous radiotherapy after previous BCT and suffered no wound complications ${ }^{(5)}$.

Postoperative complications in our study and in other series were found to be irrelevant to; pathological diagnosis, staging or age of the patient, but were found to be related to neoadjuvant chemotherapy, previous breast irradiation, thinning 
of skin flaps during dissection and to the aesthetic procedure that was adopted.

In this study, assessment of the aesthetic results and patient's satisfaction was done through patient's questionnaire by the surgical team themselves involving a number of subjective evaluations of the reconstructed breast based on a four-point ordinal scale which was established by Tzafetta's ${ }^{(\mathbf{1 4})}$. The patients were classified according to their psychological satisfaction about the reconstructive procedure into 4 groups ranging from extremely satisfied, satisfied, less satisfied and finally dissatisfied.

In Tzafetta's retrospective study of 75 patients who had SSM and IBR, he mentioned that $31 \%$ were extremely satisfied, $58.5 \%$ satisfied, $9 \%$ less satisfied and $1.5 \%$ dissatisfied $(10.5 \%$ total dissatisfaction) ${ }^{(\mathbf{1 4})}$.

The psychological benefits of immediate breast reconstruction are greater than those of delayed reconstruction and compare favorably with breast conservation therapy in psychosocial outcome. In one comparison of immediate versus delayed breast reconstruction, there was lower postoperative psychological morbidity in the former ${ }^{(15)}$.

In the current study, all patients were closely followed up to detect any signs of local recurrence (LR). The duration of postoperative follow up ranged from 24 months up to 48 months with a mean of 31.55 month of follow up. Only 1 patient $(1.2 \%)$ showed signs of local recurrence after 1 year of surgery. It was not associated with any sign of regional or systemic recurrence. This low incidence of local recurrence may be attributed to the early stage of the disease at the time of surgical intervention, the mastectomy procedure itself and the justified administration of neoadjuvant and adjuvant therapy to the studied patients. However, a relatively short period of follow up might be a drawback of obtaining a solid conclusion.

Several studies have evaluated SSM for breast cancer and found the incidence of LR is comparable to conventional mastectomy. In a 15year retrospective series of women with stage 0-2 breast cancer, 225 patients undergoing SSM and IBR were compared to 1022 patients treated by conventional mastectomy. After an average followup of 49 months, no significant difference in LR was found ${ }^{(16)}$.

After an average follow-up of 51 months, Meretoja $\boldsymbol{e t}$ al. reported only 4 local recurrences within the native breast skin of 146 women with stage $0-2$ breast cancer (2.7\% of cases) and 3 cases with isolated regional lymph nodal recurrence at a rate of $2.1 \%$. The overall loco regional recurrence was $4.8 \%$. Following surgical and oncological treatment none of those patients developed new recurrences after a mean of 35 months, suggesting that not all local recurrences are associated with disseminated disease ${ }^{(\mathbf{1 7})}$.

Recognized risk factors for LR after SSM and IBR include: tumor size, stage, poor differentiation and lymph node involvement. Uriburu $\boldsymbol{e t}$ al. also recommend surgical resection of any needle biopsy tracts at the time of SSM to reduce the risk of biopsy site LR ${ }^{(18)}$.

In a study by Gerber $\boldsymbol{e t}$ al. patients and surgeons evaluated aesthetic results of SSM versus NSM after 12 months. Patients rated satisfaction with SSM and NSM similarly with the majority ranked aesthetic outcome as good or excellent. The surgeons, however, rated $74 \%$ of NSM excellent and $26 \%$ good, while rating only $59 \%$ of SSM excellent, $22 \%$ good, and $20 \%$ fair $(\mathrm{P}=0.001)$. Secondary to improved cosmesis, NSM has gained popularity for patients requiring or choosing mastectomy. Therefore, the oncologic safety must be closely evaluated ${ }^{(\mathbf{1 9})}$.

Despite being commonly offered as an alternative to SSM, the indications for NSM have typically been identical to those for BCT. NSM has been considered safe in women with small, peripherally located tumors, without multicentricity, or for prophylactic mastectomy ${ }^{(\mathbf{2 0})}$. There is evidence that NSM is oncologically safe if performed as a prophylactic mastectomy or in patients who would, otherwise, be candidates for BCT, however, as with SSM, the additional tissue left behind may be associated with an increase in LR over conventional mastectomy for more advanced tumors with more aggressive biology. While there is data supporting the safety of SSM for larger tumors and more advanced stages, there is less applied to NSM, and additional study, preferably prospective, should be performed. The literature regarding margins for NSM focuses on the margin at the NAC, but it is prudent to remember that superficial and deep margins apply as well, and this has not been sufficiently studied or addressed ${ }^{(21)}$.

\section{CONCLUSIONS}

- Skin sparing mastectomy and Immediate breast reconstruction is a technically feasible and oncologically safe procedure.

- Accepted cosmetic result was attained in most of patients.

- Post-operative complications (mild, moderate and major) were encountered in few cases.

- Poor aesthetic results were related to: neoadjuvant chemotherapy, previous breast irradiation, thinning 
of skin flaps during mastectomy and the reconstructive procedure itself.

- Local recurrence rate was $1.25 \%$ which is comparable to its rate after non-skin sparing mastectomies.

\section{REFERENCES}

1. Howlader N, Noone AM, Krapcho M et al. (2019): SEER Cancer Statistics Review. https://seer.cancer.gov/csr/1975_2016/.

2. Omar S, Khaled H, Gaafar R et al. (2003): Breast cancer in Egypt: a review of disease prevention and detection stratigies. Eastern Mediterranean health Journal, 9(3):448-63.

3. Novak J, Besic N, Dzodic R et al. (2018): Preoperative and intra-operative detection of axillary lymph node metastases in 108 patients with invasive lobular breast cancer undergoing mastectomy. BMC Cancer, 18:137-9.

4. Garcia-Gutierrez S, Orive M, Sarasqueta C et al. (2018): Health services research in patients with breast cancer (CAMISS-prospective): study protocol for an observational prospective study. BMC Cancer, 18:54-7.

5. Reefy S, Patani N, Anderson A et al. (2010): Oncological outcome and patient satisfaction with skin-sparing mastectomy and immediate braest reconstruction: a prospective observational study. BMC cancer, 10:171-5.

6. Cunnick GH, Mokbel K (2004): Skin-sparing mastectomy. Am J Surg., 188(1):78-84.

7. Agha RA, Wellstead G, Sagoo H et al. (2016): Nipple sparing versus skin sparing mastectomy: a systematic review protocol. BMJ Open, 6(5):e010151.

8. Lam WWT, Kwong A, Suen D et al. (2018): Factors predicting patient satisfaction in women with advanced breast cancer: a prospective study. BMC Cancer, 18:162-4.

9. Simmons RM, Adamovich TL (2003): Skinsparing mastectomy. Surg Clin N Am., 83:885-899.

10. Recht A, Edge SB (2003): Evidence-based indications for postmastectomy irradiation. Surg Clin North Am., 83:995-1013.

11. Rusby J, Agha RA (2016): Nipple and breast anatomy. In Operative Approaches to Nipple-
Sparing Mastectomy, Harness JK,Willey SC (eds). Springer: Baltimore, Pp.11-19.

12. Kinoshita $S$, Nojima $K$, Takeishi $M$ et al. (2011): Retrospective Comparison of Non-Skin-Sparing Mastectomy and Skin-Sparing Mastectomy with Immediate Breast Reconstruction. International J of Surg Oncol., 10:37-49.

13. Romics L, Chew BK, Doughty JC et al. (2012): Ten-year follow-up of Skin-Sparing Mastectomy followed by Immediate Breast Reconstruction. British J of Surg., 99:799-806.

14. Tzaffeta K, Ahmed O, Bahia H et al. (2016): Evaluation of the factors related to Postmastectomy Breast Reconstruction. Plast Reconstr Surg., 107(7):1694-701.

15. Stevens LA, McGroth MH, Kister SJ et al. (1984): The psychological impact of immediate breast reconstruction for women with early breast cancer. Plast Reconstr Surg., 73(4):619-28.

16. Greenway RM, Schlossberg L, Dooley WC (2005): Fifteen-year series of skin sparing mastectomy for stage 0 to 2 breast cancer. Am J Surg., 190(6):918-22.

17. Meretoja TJ, von Smitten KA, Leidenius MH et al. (2007): Local recurrence of stage 1 and 2 breast cancer after skin-sparing mastectomy and immediate breast reconstruction in a 15-year series. Eur J Surg Oncol., 33(10):1142-5.

18. Uriburu JL, Vuoto HD, Cogorno L et al. (2006): Local recurrence of breast cancer after skin sparing mastectomy following needle core biopsy: case reports and review of the literature. Breast Journal, 12(3):194-8.

19. Gerber B, Krause A, Reimer T (2003): Skinsparing mastectomy with conservation of the nippleareola complex and autologous reconstruction is an oncologically safe procedure. Annals of Surgery, 238(1):120-7.

20. De Alcantara Filho P, Capko D, Barry JM et al. (2011): Nipple-sparing mastectomy for breast cancer and risk-reducing surgery: the memorial sloan-kettering cancer center experience. Annals of Surgical Oncology, 18(11): 3117-22.

21. Tokin CH, Weiss A, Rodriguez JW et al. (2012): Oncological Safety of Skin-Sparing and NippleSparing Mastectomy: A Discussion and Review of the Literature. https://www.ncbi.nlm.nih.gov/pubmed/22848803. 